\title{
椭圆曲线的 $K_{2}$ 群的秩
}

\author{
纪庆忠, 秦厚㭉
}

南京大学数学系, 南京 210093

E-mail: qingzhji@nju.edu.cn, hrqin@nju.edu.cn

收稿日期: 2007-12-29; 接受日期: 2008-10-27

国家自然科学基金 (批准号: 10571080, 10871088) 资助项目

摘要 本文证明了 (i) 如果定义在有理数域 $\mathbb{Q}$ 上的粗圆曲线 $E$ 的挠子群是 $N$ 阶循环 群, 且 $N \geqslant 4$, 则 $\operatorname{rank}\left(K_{2}(E)\right) \geqslant 1$; (ii) 如果定义在有理数域 $\mathbb{Q}$ 上的粗圆曲线 $E$ 的挠 子群是 3 阶循环群, 则最多除去一个 $\mathbb{R}$ - 同构类, 均有 $\operatorname{rank}\left(K_{2}(E)\right) \geqslant 1$. 同时也给出了 $\operatorname{rank}\left(K_{2}\left(E_{\mathbb{Z}}\right)\right) \geqslant 1$ 的充分条件.

关键词椭圆曲线正则映射 Néron 模

$\operatorname{MSC}(2000)$ 主题分类 $11 \mathrm{G} 05,11 \mathrm{G} 55$

\section{1 引言}

设 $E$ 是定义在复数域 $\mathbb{C}$ 上的椭圆曲线. Beilinson, Bloch 等定义了一个正则映射 $R$ : $K_{2}(\mathbb{C}(E)) \longrightarrow \mathbb{C}$. Bloch 和 Beilinson 猜测把定义在有理数域 $\mathbb{Q}$ 上的椭圆曲线 $E$ 的 $L$ - 函数 在 $s=2$ 处的值与 $K_{2}(E)$ 上的 Regulator 联系起来. 更一般的关于定义在数域 $F$ 上的椭圆 曲线 $E$ 的 $K$ 群的秩的猜测是: 对 $j>1$, $\operatorname{ord}_{s=2-j} L(E, s)=\operatorname{rank}\left(K_{2 j-2}(E)\right)=[F: \mathbb{Q}]$. 但是 $E / \mathbb{Q}$ 在具有乘法约化的素数时, 文献 [1] 中的计算表明上述猜测中 $K_{2}\left(E_{\mathbb{Q}}\right)$ 应改为 $K_{2}\left(E_{\mathbb{Z}}\right)$, 其中 $E_{\mathbb{Z}}$ 是 $E$ 的 Néron 模. Beilinson-Deligne 猜测隐含着 $\operatorname{rank}\left(K_{2}(E)\right)=1+\sharp \operatorname{Spl}(E)$, 其 中 $\operatorname{Spl}(E)$ 是 $E$ 的具有可分乘法约化的素数集合. Grayson 对文献 [2] 中的 Swinnerton-Dyer 表中的导子 $>180$, 具有负的判别式, 而且具有阶不小于 5 的有理点的所有椭圆曲线 (共 37 条) $K_{2}$ 群的秩进行了数值计算. Ross 在文献 [3] 中证明了对于定义在有理数域 $\mathbb{Q}$ 上并且至 少拥有一个 3 阶有理点的椭圆曲线 $E$, 除去有限个 $\mathbb{R}$ - 同构类外, 均有 $\operatorname{rank}\left(K_{2}(E)\right) \geqslant 1$. 本 文证明了: 对所有定义在有理数域 $\mathbb{Q}$ 上并且拥有一个阶至少为 4 的有理点的椭圆曲线 $E$, 均有 $\operatorname{rank}\left(K_{2}(E)\right) \geqslant 1$; 如果 $E / \mathbb{Q}$ 拥有一个 3 阶有理点, 则最多除一个 $\mathbb{R}-$ 同构类外, 均有 $\operatorname{rank}\left(K_{2}(E)\right) \geqslant 1$. 也给出 $\operatorname{rank}\left(K_{2}\left(E_{\mathbb{Z}}\right)\right) \geqslant 1$ 的充分条件. 同时我们也给出 $\operatorname{rank}\left(K_{2}\left(E_{\mathbb{Z}}\right)\right) \geqslant 1$ 和 $\operatorname{rank}\left(K_{2}(E)\right) \geqslant 1+\sharp \operatorname{Spl}(E)$ 的例子. 如果 $m \geqslant 4$, 令 $E[m]$ 表示 $E(\mathbb{C})$ 的所有 $m$ 阶点生成 的子群, 令 $k=\mathbb{Q}(E[m])$. 我们证明了 $K_{2}\left(E_{k}\right)$ 的秩至少是 2 .

\section{2 预备知识}

$$
\text { 设 } r, s, x, y \in \mathbb{R} \text {, 且 } y>0 \text {. 令 } \tau=x+y i \text {, 定义 }
$$




$$
\varepsilon(r, s, \tau)=\Sigma^{\prime}(m \tau+n)|m \tau+n|^{-4} e^{2 \pi i(m r+n s)},
$$

其中 $m, n$ 遍历所有的整数, 且 $(m, n) \neq(0,0)$.

易证 $\varepsilon(r, s, \tau)$ 具有下列性质.

引理 2.1 (i) $\varepsilon(r, s, \tau)$ 只依赖于 $r$ 和 $s$ 模 $\mathbb{Z}$ 的剩余类.

(ii) $\varepsilon(-r,-s, \tau)=-\varepsilon(r, s, \tau)$. 因此

(iii) 如果

$$
\varepsilon(0,0, \tau)=\varepsilon\left(\frac{1}{2}, 0, \tau\right)=\varepsilon\left(0, \frac{1}{2}, \tau\right)=\varepsilon\left(\frac{1}{2}, \frac{1}{2}, \tau\right)=0 .
$$

$$
\gamma=\left(\begin{array}{ll}
a & b \\
c & d
\end{array}\right) \in S L_{2}(\mathbb{Z})
$$

则

$$
\varepsilon(r, s, \gamma \tau)=\frac{|c \tau+d|^{4}}{c \tau+d} \varepsilon(d r-b s, a s-c r, \tau) .
$$

令 $\mathbb{H}$ 表示上半平面, 设 $\tau \in \mathbb{H}$. $E$ 表示由格 $\Lambda_{\tau}=\mathbb{Z}+\mathbb{Z} \tau$ 决定的椭圆曲线. Beilinson 定 义了正则映射 $R_{\tau}: K_{2}(\mathbb{C}(E)) \longrightarrow \mathbb{C}$ 并且给出 $R_{\tau}(\{f, g\})$ 的计算公式.

引理 $2.2^{[4]}$ 设 $\tau \in \mathbb{H}, E$ 是定义在复数域 $\mathbb{C}$ 上的椭圆曲线, 并且 $E \cong \mathbb{C} / \Lambda_{\tau}$. 若将 $z \in \mathbb{C} / \Lambda_{\tau}$ 表示成 $z=v(z)+u(z) \tau\left(\bmod \Lambda_{\tau}\right)$, 其中 $v(z), u(z) \in[0,1)$, 设 $f, g \in \mathbb{C}(E)^{*}$, 则

$$
R_{\tau}(\{f, g\})=\frac{(\operatorname{Im} \tau)^{2}}{\pi^{2}} \sum_{z, w \in \mathbb{C} / \Lambda_{\tau}}\left(\operatorname{order}_{z}(f)\right)\left(\operatorname{order}_{w}(g)\right) \varepsilon(v(z-w),-u(z-w), \tau) \text {. }
$$

设 $E$ 是定义在数域 $k$ 上的椭圆曲线, 则有正合局部化序列 (参见文献 [5])

$$
K_{2}(E) \longrightarrow K_{2}(k(E)) \stackrel{\text { tame }}{\longrightarrow} \coprod_{x \in E} k(x)^{*},
$$

其中左边箭头的核是挠子群, 因此如果要得到 $K_{2}(E)$ 中的一个无限阶元素, 只须在映射 tame 的核中构造一个元素. 正则映射 $R_{E}$ 限制在 $K_{2}(E)$ 上便得到 $K_{2}(E)$ 上的一个正则映射. 另 一方面, 设 $c \in \mathbb{C}^{*}$, 则 $R_{E}$ 作用在符号 $\{f, c\}$ 上是平凡的. 因此只需考虑 $K_{2}(k(E))$ 模由 $\{f, c\}$ 生成的子群的剩余类, 其中 $c \in \mathbb{C}^{*}$.

引理 $2.3^{[5]}$ 设 $E$ 是定义在数域 $k$ 上的椭圆曲线. $N$ 是一个正整数, 并且所有 $N$ 阶点 生成的群 $E[N] \subset E(k)$. 设 $f, g \in k(E)$ 并且 $\operatorname{support}(\operatorname{div}(f)), \operatorname{support}(\operatorname{div}(g)) \subset E[N]$, 则存在 $f_{i} \in k(E)^{*}, c_{i} \in k^{*}$, 使得

$$
\{f, g\}^{N} \prod\left\{f_{i}, c_{i}\right\} \in \operatorname{Ker}(\text { tame symbol). }
$$

注记 1 设 $k$ 是一个数域, $E / k$ 是一条椭圆曲线, $P \in E(k)$ 是阶为 $N \geqslant 3$ 的 $k$-有理点. 对于任意的 $1 \leqslant s \leqslant N-1$, 令 $g_{P}, f_{P, s} \in k(E)$, 并且有 $\operatorname{div}\left(g_{P}\right)=\sum_{i=1}^{N-1} 2([i] P)-2(N-1)(O)$ 和 $\operatorname{div}\left(f_{P, s}\right)=N([s] P)-N(O)$. 定义 $S_{P, s} \in K_{2}(E)$ 如下:

$$
S_{P, s} \equiv\left\{g_{P}, f_{P, s}\right\} \text { (modulo image } K(E)^{*} \otimes k^{*} \text { ). }
$$

易证 $S_{P, s}$ 在相差一个有限阶元素的情况下是惟一决定的.

引理 2.4 设 $r, s \in[0,1)$, 而 $\tau=x+i y \in \mathbb{H}$. 则

$$
\begin{aligned}
\operatorname{Re} \varepsilon(r, s, \tau)= & \frac{i \pi}{2} y^{-2} \sum_{m \neq 0} \sum_{n} \frac{1}{m|m|} e^{2 \pi i[m(r-s x)+m n x+i y|n-s||m|]} \\
& +i \pi^{2} y^{-1} \sum_{m \neq 0} \sum_{n} \frac{|n-s|}{m} e^{2 \pi i[m(r-s x)+m n x+i y|n-s||m|]},
\end{aligned}
$$


并且

$$
\operatorname{Im} \varepsilon(r, s, \tau)=4 \pi^{3} B(s)-\pi^{2} y^{-1} \sum_{m \neq 0} \sum_{n} \frac{n-s}{|m|} e^{2 \pi i[m(r-s x)+m n x+i y|n-s||m|]},
$$

其中 $B(s)=\frac{1}{3} s^{3}-\frac{1}{2} s^{2}+\frac{1}{6} s, s \in[0,1)$.

证明 由 (1) 式, 有

$$
\begin{aligned}
\varepsilon(r, s, \tau) & =\Sigma^{\prime}(m \tau+n)|m \tau+n|^{-4} e^{2 \pi i(m r+n s)} \\
& =\tau \Sigma^{\prime} m|m \tau+n|^{-4} e^{2 \pi i(m r+n s)}+\Sigma^{\prime} n|m \tau+n|^{-4} e^{2 \pi i(m r+n s)} \\
& =\tau f(\tau)+g(\tau)+4 \pi^{3} i B(s),
\end{aligned}
$$

其中

$$
\begin{aligned}
& f(\tau)=\sum_{m \neq 0} \sum_{n} m|m \tau+n|^{-4} e^{2 \pi i(m r+n s)}, \\
& g(\tau)=\sum_{m \neq 0} \sum_{n} n|m \tau+n|^{-4} e^{2 \pi i(m r+n s)},
\end{aligned}
$$

并且 $B(s)=\frac{1}{3} s^{3}-\frac{1}{2} s^{2}+\frac{1}{6} s$, 具有如下 Fourier 展开式

$$
B(s)=-i \frac{1}{4 \pi^{3}} \sum_{n \neq 0} \frac{e^{2 \pi i n s}}{n^{3}}, \quad s \in[0,1) .
$$

令

$$
\begin{aligned}
& f(\tau, z)=\sum_{m \neq 0} \sum_{n} \frac{m}{|m \tau+n|^{4 z}} e^{2 \pi i(m r+n s)}, \\
& g(\tau, z)=\sum_{m \neq 0} \sum_{n} \frac{n}{|m \tau+n|^{4 z}} e^{2 \pi i(m r+n s)}, \\
& F(\tau, z)=\sum_{m \neq 0} \sum_{n} \frac{1}{m}|m \tau+n|^{2-4 z} e^{2 \pi i(m r+n s)} .
\end{aligned}
$$

由文献 [3] 中引理 2.2 知, $F(\tau, 1)=\pi y^{-1} T(\tau)$, 其中

$$
T(\tau)=\sum_{m \neq 0} \sum_{n} \frac{1}{m|m|} e^{2 \pi i[m(r-s x)+m n x+i y|n-s||m|]} .
$$

另一方面, 有

$$
\begin{aligned}
& \frac{\partial F(\tau, z)}{\partial y}=2 y(1-2 z) f(\tau, z), \\
& \frac{\partial F(\tau, z)}{\partial x}=2(1-2 z)[x f(\tau, z)+g(\tau, z)] .
\end{aligned}
$$

又

$$
\begin{aligned}
\frac{\partial F(\tau, 1)}{\partial x}=\pi y^{-1} & \frac{\partial T}{\partial x}=i 2 \pi^{2} y^{-1} \sum_{m \neq 0} \sum_{n} \frac{n-s}{|m|} e^{2 \pi i[m(r-s x)+m n x+i y|n-s||m|]}, \\
\frac{\partial F(\tau, 1)}{\partial y}= & -\pi y^{-2} T(\tau)+\pi y^{-1} \frac{\partial T}{\partial y} \\
= & -\pi y^{-2} \sum_{m \neq 0} \sum_{n} \frac{1}{m|m|} e^{2 \pi i[m(r-s x)+m n x+i y|n-s||m|]} \\
& -2 \pi^{2} y^{-1} \sum_{m \neq 0} \sum_{n} \frac{|n-s|}{m} e^{2 \pi i[m(r-s x)+m n x+i y|n-s \|| m \mid]}
\end{aligned}
$$


因此

$$
\begin{aligned}
\varepsilon(r, s, \tau)= & \tau f(\tau)+g(\tau)+4 \pi^{3} B(s) i \\
= & (x+i y)\left(-\frac{1}{2 y} \frac{\partial F(\tau, 1)}{\partial y}\right)-\frac{1}{2} \frac{\partial F(\tau, 1)}{\partial x}-x\left(-\frac{1}{2 y} \frac{\partial F(\tau, 1)}{\partial y}\right)+4 \pi^{3} B(s) i \\
= & -\frac{i}{2} \frac{\partial F(\tau, 1)}{\partial y}-\frac{1}{2} \frac{\partial F(\tau, 1)}{\partial x}+4 \pi^{3} B(s) i \\
= & i \frac{\pi y^{-2}}{2} \sum_{m \neq 0} \sum_{n} \frac{1}{m|m|} e^{2 \pi i[m(r-s x)+m n x+i y|n-s||m|]} \\
& +i \pi^{2} y^{-1} \sum_{m \neq 0} \sum_{n} \frac{|n-s|}{m} e^{2 \pi i[m(r-s x)+m n x+i y|n-s||m|]} \\
& -i \pi^{2} y^{-1} \sum_{m \neq 0} \sum_{n} \frac{n-s}{|m|} e^{2 \pi i[m(r-s x)+m n x+i y|n-s||m|]} \\
& +i 4 \pi^{3} B(s) .
\end{aligned}
$$

所以

$$
\begin{aligned}
\operatorname{Re} \varepsilon(r, s, \tau)= & \frac{i \pi}{2} y^{-2} \sum_{m \neq 0} \sum_{n} \frac{1}{m|m|} e^{2 \pi i[m(r-s x)+m n x+i y|n-s||m|]} \\
& +i \pi^{2} y^{-1} \sum_{m \neq 0} \sum_{n} \frac{|n-s|}{m} e^{2 \pi i[m(r-s x)+m n x+i y|n-s||m|]},
\end{aligned}
$$

并且

$$
\operatorname{Im} \varepsilon(r, s, \tau)=4 \pi^{3} B(s)-\pi^{2} y^{-1} \sum_{m \neq 0} \sum_{n} \frac{n-s}{|m|} e^{2 \pi i[m(r-s x)+m n x+i y|n-s||m|]} .
$$

由于我们的研究对象是定义在有理数域上的椭圆曲线, 因此要用到下面的关于定义在实 数域上的椭圆曲线的性质.

引理 2.5 设 $E$ 是定义在实数域 $\mathbb{R}$ 上的一条椭圆曲线, $\Delta(E)$ 是 $E$ 的判别式. 则

(a) 存在惟一的 $\tau \in \mathbb{J}=\{i t: t>0\} \cup\left\{\frac{1}{2}+i t: t>0\right\}$, 使得

$$
\psi: \mathbb{C} / \Lambda_{\tau} \longrightarrow E(\mathbb{C})
$$

同构, 并且与复共轭可交换, 也就是说, $\psi$ 是定义在 $\mathbb{R}$ 上的同构.

(b) (参见文献 [6] 中第五章, 推论 2.3.1) 存在下列实 Lie 群的同构:

$$
E(\mathbb{R}) \cong \begin{cases}\mathbb{R} / \mathbb{Z}, & \text { 若 } \Delta(E)<0, \\ (\mathbb{R} / \mathbb{Z}) \times(\mathbb{Z} / 2 \mathbb{Z}), & \text { 若 } \Delta(E)>0 .\end{cases}
$$

\section{3 主要结果}

设 $E$ 是一条定义在复数域 $\mathbb{C}$ 上的椭圆曲线, 则存在 $\tau \in \mathbb{H}$, 使得

$$
\varphi: \mathbb{C} / \Lambda_{\tau} \longrightarrow E(\mathbb{C})
$$

是同构. 假设 $P \in E(\mathbb{C})$ 是一个 $N$ 阶点. 由引理 2.3 后的注记 1 知, 对于 $1 \leqslant s \leqslant N-1$, 函 数 $g_{P}, f_{P, s} \in \mathbb{C}(E)$ 以及 $S_{P, s} \in K_{2}(E)$ 是存在的. 如果 $P$ 在同构 $\varphi$ 下对应于 $\frac{a}{N}+\frac{b}{N} \tau$, 则 $R_{E}\left(S_{P, s}\right)$ 的计算公式由下面的定理给出.

定理 $3.1 R_{E}\left(S_{\frac{a}{N}+\frac{b}{N} \tau, s}\right)=-2 \frac{N^{2}(\operatorname{Im} \tau)^{2}}{\pi^{2}} \varepsilon\left(-\frac{s a}{N}, \frac{s b}{N}, \tau\right)$. 
证明 由引理 2.2 , 得

$$
\begin{aligned}
R_{E}\left(S_{\frac{a}{N}+\frac{b}{N} \tau, s}\right)= & R_{E}\left(\left\{g_{P}, f_{P, s}\right\}\right) \\
= & \frac{(\operatorname{Im} \tau)^{2}}{\pi^{2}}\left[2 N \sum_{i=1}^{N-1} \varepsilon\left(\frac{i a-s a}{N},-\frac{i b-s b}{N}, \tau\right)-2 N \sum_{i=1}^{N-1} \varepsilon\left(\frac{i a}{N},-\frac{i b}{N}, \tau\right)\right. \\
& \left.\quad-2 N(N-1) \varepsilon\left(\frac{-s a}{N},-\frac{-s b}{N}, \tau\right)+2 N(N-1) \varepsilon(0,0, \tau)\right] \\
= & -\frac{2 N^{2}(\operatorname{Im} \tau)^{2}}{\pi^{2}} \varepsilon\left(\frac{-s a}{N}, \frac{s b}{N}, \tau\right) .
\end{aligned}
$$

由引理 2.2 和定理 3.1 , 即可得到 $K_{2}(E)$ 中一些元素在正则映射下的像之间的 $\mathbb{Z}$ - 线形 关系.

推论 3.2 设 $E / \mathbb{C}$ 是一条椭圆曲线, $N$ 是正整数. 如果函数 $F, G \in \mathbb{C}(E)^{*}$ 的支撑点都 是 $E(\mathbb{C})$ 的 $N$ 阶点, 则 $2 N^{2} R_{E}(\{F, G\})$ 是一些 $R_{E}\left(S_{P, s}\right)$ 的 $\mathbb{Z}$ - 线性组合, 其中 $P \in E[N]$, $1 \leqslant s \leqslant N-1$.

注记 2 设 $k$ 是数域, $E / k$ 是一条椭圆曲线. 设函数 $F, G \in k(E)^{*}$ 的支撑点都是 $E(k)$ 的 $N$ 阶点, 则存在元素 $S_{F, G} \in K_{2}(E)$, 使得

$$
S_{F, G} \equiv\{F, G\} \text { (modulo image } k(E)^{*} \otimes k^{*} \text { ). }
$$

引理 2.2 和推论 3.2 隐含着 $2 N^{2} R_{E}\left(S_{F, G}\right)$ 是一些 $R_{E}\left(S_{P, s}\right)$ 的线性组合, 其中 $1 \leqslant s \leqslant N-1$, $P \in E[N](k)$.

由定理 3.1 知, 要分析 $R_{E}\left(S_{\frac{a}{N}+\frac{b}{N} \tau, s}\right)$ 只需计算 $\varepsilon\left(-\frac{s a}{N}, \frac{s b}{N}, \tau\right)$. 下面的几个引理分各种情 形分析 $\varepsilon\left(-\frac{s a}{N}, \frac{s b}{N}, \tau\right)$, 为了更快地得到我们的主要结果, 将这些需要一些技巧的引理的证明 放在第 4 节.

引理 3.3 对任意的 $r \in\left(0, \frac{1}{2}\right)$ 和 $y>0$, 则 $\varepsilon(r, 0, i y)$ 是一个负实数.

引理 3.4 对任意的 $y>0$, 则 $\varepsilon\left(\frac{1}{4}, \frac{1}{2}, i y\right)$ 是一个负实数.

引理 3.5 对任意的 $y>0, r \in\left(0, \frac{1}{4}\right]$ 和 $\tau=\frac{1}{2}+i y$, 则 $\varepsilon(r, 0, \tau)$ 是一个负实数.

引理 $3.6 \varepsilon\left(\frac{1}{3}, 0, \frac{1}{2}+i y\right)$ 在区间 $(0, \infty)$ 内只有一个零点.

推论 3.7 设 $k$ 是一个数域, $E / k$ 是一条椭圆曲线, $P \in E(k)$ 是一个有限阶元素, 其阶 为 $N$, 对于 $1 \leqslant s \leqslant N-1$, 由注记 1 有元素 $S_{P, s} \in K_{2}(E)$. 则 $R_{E}\left(S_{P, s}\right)$ 是一个实数.

由上面的一些引理, 可得到我们的主要结果.

定理 3.8 设 $E / \mathbb{Q}$ 是一条椭圆曲线, 并且 $E(\mathbb{Q})$ 包含一个阶为 $N$ 的有限阶点.

(a) 如果 $N \geqslant 4$, 则 $K_{2}(E)$ 至少包含一个无限阶元素, 即 $K_{2}(E)$ 具有正的秩.

(b) 假设 $N=3$.

(i) 如果 $\Delta(E)>0$, 则 $K_{2}(E)$ 具有正的秩.

(ii) 如果 $\Delta(E)<0$, 则最多除去一个 $\mathbb{R}$ - 同构类外, $K_{2}(E)$ 具有正的秩.

证明 由引理 2.5 知, 存在惟一的 $\tau \in \mathbb{J}$, 其中

$$
\mathbb{J}=\{i t: t>0\} \cup\left\{\frac{1}{2}+i t: t>0\right\},
$$

使得 $\psi: \mathbb{C} / \Lambda_{\tau} \longrightarrow E$ 是定义在 $\mathbb{R}$ 上的同构, 其中 $\Lambda_{\tau}=\mathbb{Z}+\mathbb{Z} \tau$.

(I) 如果 $y>0$, 且 $\tau=y i$, 那么 $E(\mathbb{R})$ 有两个分支. 
设 $P \in E(\mathbb{Q})$ 是一个有限阶点且阶为 $N$, 则 $P$ 在同构 $\psi$ 下对应于 $\frac{a}{N}+t i y$, 其中 $t=0$ 或 $\frac{1}{2}$ 并且 $(a, N)=1$.

(1) 假设 $t=0$. 由引理 2.1 和引理 3.3 得

$$
R_{E}\left(S_{P, 1}\right)=-2 \frac{N^{2} y^{2}}{\pi^{2}} \varepsilon\left(\frac{N-a}{N}, 0, i y\right) \neq 0 .
$$

(2) 假设 $t=\frac{1}{2}$, 且 $N \neq 4$. 则 $\frac{2 a}{N} \neq \frac{1}{2}$, 因此由引理 2.1 和引理 3.3 得

$$
R_{E}\left(S_{2 P, 1}\right)=-2 \frac{N^{2} y^{2}}{\pi^{2}} \varepsilon\left(\frac{N-2 a}{N}, 0, i y\right) \neq 0 .
$$

(3) 假设 $t=\frac{1}{2}, N=4$, 令 $P$ 在同构 $\psi$ 下对应于 $\frac{1}{4}+\frac{i y}{2}$. 由引理 3.4 , 对任意 $y>0$, 有

$$
R_{E}\left(S_{P, 1}\right)=-2 \frac{N^{2} y^{2}}{\pi^{2}} \varepsilon\left(\frac{3}{4}, \frac{1}{2}, i y\right)=2 \frac{N^{2} y^{2}}{\pi^{2}} \varepsilon\left(\frac{1}{4}, \frac{1}{2}, i y\right) \neq 0 .
$$

(II) 如果 $y>0$, 且 $\tau=\frac{1}{2}+i y$, 那么 $E(\mathbb{R})$ 有一个分支.

设 $P \in E(\mathbb{Q})$ 是一个有限阶点且阶为 $N$, 则 $P$ 在同构 $\psi$ 下对应于 $\frac{N-1}{N}$.

(1) 假设 $N \geqslant 4$, 由引理 3.5 得

$$
R_{E}\left(S_{P, 1}\right)=-2 \frac{N^{2} y^{2}}{\pi^{2}} \varepsilon\left(\frac{1}{N}, 0, \frac{1}{2}+i y\right) \neq 0 .
$$

(2) 假设 $N=3$, 由引理 3.6 , 最多除去一个点 $y=y_{0}>0$, 对其他任意的 $y>0$, 都有

$$
R_{E}\left(S_{P, 1}\right)=-2 \frac{3^{2} y^{2}}{\pi^{2}} \varepsilon\left(\frac{1}{3}, 0, \frac{1}{2}+i y\right) \neq 0 .
$$

又对于 $y>0$, 在 $\mathbb{R}$ - 同构: $E \longrightarrow \mathbb{C} / \Lambda_{\tau}$ 之下, $\Delta(E)>0$ (或 $\Delta(E)<0$ ), 当且仅当 $\tau=i y$ (或 $\tau=\frac{1}{2}+i y$ ). 从而证明了定理 3.8 .

定理 3.9 设 $E / \mathbb{Q}$ 是一条椭圆曲线. 正整数 $N \geqslant 3$, 并令 $E[N]$ 表示 $E(\mathbb{C})$ 中所有 $N$ 阶点生成的子群, 令 $k=\mathbb{Q}(E[N])$.

(1) 如果 $\Delta(E)>0$ 且 $N \geqslant 3$, 则 $\operatorname{rank}\left(K_{2}\left(E_{k}\right)\right) \geqslant 2$;

(2) 如果 $\Delta(E)<0$ 且 $N \geqslant 4$, 则 $\operatorname{rank}\left(K_{2}\left(E_{k}\right)\right) \geqslant 2$.

证明 由引理 2.5 知存在 $\tau \in \mathbb{J}$, 使得 $\psi: \mathbb{C} / \Lambda_{\tau} \longrightarrow E$ 是 $\mathbb{R}$-同构. 令 $P_{1}$ 是在 $\mathbb{R}$ - 同构 $\psi$ 下对应于 $\frac{1}{N}$ 的 $N$ 阶点.

(i) 如果 $\Delta(E)>0$, 那么 $\operatorname{Re} \tau=0$. 令 $P_{2}$ 是在 $\mathbb{R}$ - 同构 $\psi$ 下对应于 $\frac{1}{N} \tau$ 的 $N$ 阶点. 如果 $N \geqslant 3$, 则由引理 3.3 知, $R_{E}\left(S_{P_{1}, 1}\right)$ 是一个非零实数, 并且 $R_{E}\left(S_{P_{2}, 1}\right)$ 是一个非零的纯虚数.

(ii) 如果 $\Delta(E)<0$, 则 $\operatorname{Re} \tau=\frac{1}{2}$. 令 $P_{2}$ 在 $\mathbb{R}-$ 同构 $\psi$ 下对应于 $-\frac{1}{N}+\frac{2}{N} \tau$. 如果 $N \geqslant 4$, 则由引理 3.5 知, $R_{E}\left(S_{P_{1}, 1}\right)$ 是一个非零实数, 并且 $R_{E}\left(S_{P_{2}, 1}\right)$ 是一个非零的纯虚数.

因此无论 $\Delta(E)>0$ 还是 $\Delta(E)<0, S_{P_{1}, 1}$ 和 $S_{P_{2}, 1}$ 都没有 $\mathbb{Z}$-线性关系, 从而 $\operatorname{rank}\left(K_{2}\left(E_{k}\right)\right)$ $\geqslant 2$.

设 $E / \mathbb{Q}$ 是一条椭圆曲线并且 $E(\mathbb{Q})_{\text {tors }} \cong \mathbb{Z} / N \mathbb{Z}$. 在文献 [1] 中, 大量的计算说明 $K_{2}\left(E_{\mathbb{Q}}\right)$ 的秩应该严格大于 1 , 例如椭圆曲线 $26 \mathrm{D}$ (见文献 $[1,2]$ ) 的秩应该为 2 .

如果 $N \geqslant 3$. 令 $E_{\mathbb{Z}}$ 表示 $E$ 的 Néron 模并且 $E_{p}$ 表示 $E$ 在素数 $p$ 处的特殊纤维. 如果 $E_{p}$ 是 Kodaira 型 $I_{v}(v \geqslant 1)$ 坏的纤维, 并且具有 $n$ 个边, 连续标号 $0,1, \ldots, n-1$ 使得 0 对应于与 0 - 截面相交的分支. 令 $P \in E(\mathbb{Q})_{\text {tors }}$ 是一个 $N$ 阶点. 假设 $P$ 在第 $r$ 个分支并记 $t=r / n$. 对于 $a \in \mathbb{Q}^{+},\{a\}$ 表示 $a$ 的小数部分. 
对于 $1 \leqslant s \leqslant N-1$, 令

$$
f_{p}(P, s)=\sum_{l=1}^{N-1}(\{t s\}-\{t l\})(-\{t s\}\{t l\}+\min (\{t s\},\{t l\})) .
$$

考虑局部化序列:

$$
K_{2}\left(E_{\mathbb{Z}}\right) \stackrel{\lambda}{\longrightarrow} K_{2}\left(E_{\mathbb{Q}}\right) \stackrel{\amalg \partial_{p}}{\longrightarrow} \coprod_{p} K_{1}^{\prime}\left(E_{p}\right) .
$$

由文献 [1], 有

(i) 对于具有好的约化素数 $p, K_{1}^{\prime}\left(E_{p}\right)$ 是挠子群.

(ii) 如果 $E_{p}$ 是 Kodaira 型 $I_{v}(v \geqslant 1)$ 坏的纤维, 则

$$
K_{1}^{\prime}\left(E_{p}\right) \cong \mathbb{Z} \oplus \text { torsion. }
$$

(iii) 如果 $E_{p}$ 是其他型的坏纤维, 则 $K_{1}^{\prime}\left(E_{p}\right)$ 是挠子群.

(iv) 对于纤维 $I_{v}, v \geqslant 1$, 有

$$
\partial_{p}\left(S_{P, s}\right)=(*) f_{p}(P, s),
$$

其中 $(*)$ 是一个与 $s$ 无关的常数.

(v) 在相差挠元素的情况下, 一个元素

$$
\sum_{s=1}^{\left[\frac{N-1}{2}\right]} a_{s} S_{P, s}, \quad a_{s} \in \mathbb{Z},
$$

来自于 $K_{2}\left(E_{\mathbb{Z}}\right)$, 当且仅当对所有具有乘法约化的素数 $p$ 均有

$$
\sum_{s=1}^{\left[\frac{N-1}{2}\right]} a_{s} f_{p}(P, s)=0 .
$$

由以上讨论很容易证明下面的定理.

定理 3.10 设 $N \geqslant 3, E / \mathbb{Q}$ 是一条椭圆曲线并且 $E(\mathbb{Q})_{\text {tors }} \cong \mathbb{Z} / N \mathbb{Z}$. 令 $P \in E(\mathbb{Q})_{\text {tors }}$ 在 $\mathbb{R}$ - 同构: $E \longrightarrow \mathbb{C} / \Lambda_{\tau}$ 下对应于 $\frac{1}{N}$.

(A) 如果至少存在两个非零的整数 $a_{s} \in \mathbb{Z}, 1 \leqslant s \leqslant\left[\frac{N-1}{2}\right]$ 使得

(i) 对所有的具有乘法约化的素数 $p$, 均有 $\sum_{s=1}^{\left[\frac{N-1}{2}\right]} a_{s} f_{p}(P, s)=0$ 成立;

(ii) $R_{E}\left(\sum_{s=1}^{\left[\frac{N-1}{2}\right]} a_{s} S_{P, s}\right) \neq 0$;

(iii) 存在某个 $s$ 以及有乘法约化的素数 $p$, 使得 $f_{p}(P, s) \neq 0$ 成立, 则 $\operatorname{rank}\left(K_{2}(E)\right) \geqslant 2$.

(B) 如果 $a_{i} \in \mathbb{Z}, i=1, \ldots, r, 1 \leqslant r \leqslant\left[\frac{N-1}{2}\right]$ 使得对所有的具有乘法约化的素数 $p$, 均有 $\sum_{i=1}^{r} a_{i} f_{p}\left(P, s_{i}\right)=0$ 成立, 则 $a_{i}=0, i=1, \ldots, r$. 那么 $\operatorname{rank}\left(K_{2}(E)\right) \geqslant r$.

注记 3 在文献 [1] 中, Bloch 和 Grayson 猜测 $\operatorname{rank}\left(K_{2}\left(E_{\mathbb{Z}}\right)\right)=1$. 如果存在 $\alpha \in K_{2}\left(E_{\mathbb{Z}}\right)$, 使得 $R_{E}(\alpha) \neq 0$, 则 $\operatorname{rank}\left(K_{2}\left(E_{\mathbb{Z}}\right)\right) \geqslant 1$. 由引理 3.3 和 3.5 , 即得到下面的定理.

定理 3.11 设 $N \geqslant 3, E / \mathbb{Q}$ 是一条椭圆曲线并且 $E(\mathbb{Q})_{\text {tors }} \cong \mathbb{Z} / N \mathbb{Z}$. 令 $P \in E(\mathbb{Q})_{\text {tors }}$ 在 $\mathbb{R}$-同构: $E \longrightarrow \mathbb{C} / \Lambda_{\tau}$ 下对应于 $\frac{1}{N}$. 如果 $E$ 还满足下面的两个条件:

(i) 存在整数 $a_{i} \geqslant 0$ (或 $a_{i} \leqslant 0$ ), $1 \leqslant i \leqslant\left[\frac{N-1}{2}\right]$, 并且至少有一个 $a_{i} \neq 0$, 使得对所有的 具有乘法约化的素数 $p$, 均有下式成立:

$$
\sum_{i=1}^{\left[\frac{N-1}{2}\right]} a_{i} f_{p}\left(P, s_{i}\right)=0
$$


(ii) (a) $\Delta(E)>0, N \geqslant 3$ 或 (b) $\Delta(E)<0$ 并且如果 $a_{i} \neq 0$, 则有 $\frac{s_{i}}{N} \leqslant \frac{1}{4}$.

则 $\operatorname{rank}\left(K_{2}\left(E_{\mathbb{Z}}\right)\right) \geqslant 1$.

例 1 令 $E$ 是文献 $[1,2]$ 中的椭圆曲线 $30 \mathrm{~A}$, 其 Weierstrass 方程为:

$$
E: y^{2}+x y+y=x^{3}+x+2 \text {. }
$$

$E$ 的判别式为 $\Delta(E)=-2^{4} 3^{3} 5$, 并且 $E(\mathbb{Q})_{\text {tors }}$ 是 6 阶循环群. 因此存在 $P \in E(\mathbb{Q})_{\text {tors }}$ 在 $\mathbb{R}$ - 同构: $E \longrightarrow \mathbb{C} / \Lambda_{\tau}$ 下对应于 $\frac{1}{6}$. 椭圆曲线 $E$ 在素数 $p=2,3,5$ 处的 Kodaira 型分别为 $I_{4}, I_{3}, I_{1}$. 由于 $\partial_{5}=0$, 只需对 $s=1,2$ 时计算 $f_{2}(P, s)$ 和 $f_{3}(P, s)$. 事实上, 我们有

(i) $f_{2}(P, 1)=f_{2}(P, 2)=0, f_{3}(P, 1)=-\frac{2}{27}=-f_{3}(P, 2)$;

(ii) $R_{E}\left(S_{P, 1}+S_{P, 2}\right) \neq 0$.

由于 $\operatorname{Spl}(E)=\{3\}$, 因此由定理 3.10 得

$$
\operatorname{rank}\left(K_{2}(E)\right) \geqslant 2=1+\sharp \operatorname{Spl}(E) .
$$

由注记 3 得

$$
\operatorname{rank}\left(K_{2}\left(E_{\mathbb{Z}}\right)\right) \geqslant 1 .
$$

因此如果假设 Beilinson-Deligne 猜测成立, 则我们得到 $K_{2}(E) \otimes \mathbb{Q}$ 的一组基.

例 2 令 $E$ 是文献 [7] 中的椭圆曲线 $870 I_{2}$, 其 Weierstrass 方程为:

$$
y^{2}+x y=x^{3}-43360 x+3450272 .
$$

$E$ 的判别式 $\Delta(E)=2^{5} 3^{5} 5^{10} 29^{2}>0$ 并且 $E(\mathbb{Q})_{\text {tors }} \cong \mathbb{Z} / 10 \mathbb{Z}$. 因此存在 $P \in E(\mathbb{Q})_{\text {tors }}$ 在 $\mathbb{R}-$ 同构: $E \longrightarrow \mathbb{C} / \Lambda_{\tau}$ 下对应于 $\frac{1}{10}$. 椭圆曲线 $E$ 在素数 $p=2,3,5,29$ 处的 Kodaira 型分别为 $I_{5}, I_{5}, I_{10}, I_{2}$. 我们很容易得到

(i) $f_{2}(P, 1)=-\frac{4}{25}, f_{2}(P, 2)=-\frac{2}{25}, f_{2}(P, 3)=\frac{2}{25}, f_{2}(P, 4)=\frac{4}{25}$;

(ii) $f_{3}(P, 1)=-\frac{2}{25}, f_{3}(P, 2)=\frac{4}{25}, f_{3}(P, 3)=-\frac{4}{25}, f_{3}(P, 4)=\frac{2}{25}$;

(iii) $f_{5}(P, 1)=-\frac{7}{50}, f_{5}(P, 2)=\frac{2}{25}, f_{5}(P, 3)=\frac{3}{25}, f_{5}(P, 4)=-\frac{4}{25}$;

(iv) $f_{29}(P, s)=0,1 \leqslant s \leqslant 4$.

因此

(1) $S_{P, 1}, S_{P, 2}, S_{P, 3}$ 在 $K_{2}(E)$ 中 $\mathbb{Z}$-线性无关.

(2) $E$ 在素数 $p=2,3,5,29$ 处具有乘法约化, 并且

$$
2 f_{p}(P, 1)+3 f_{p}(P, 2)+3 f_{p}(P, 3)+2 f_{p}(P, 4)=0 .
$$

由于 $\operatorname{Spl}(E)=\{2,3,5\}$, 所以

$$
\operatorname{rank}\left(K_{2}(E)\right) \geqslant 4=1+\sharp \operatorname{Spl}(E), \text { 而 } \operatorname{rank}\left(K_{2}\left(E_{\mathbb{Z}}\right)\right) \geqslant 1 .
$$

与例 1 一样, 如果假设 Beilinson-Deligne 猜测成立, 则

$$
\operatorname{rank}\left(K_{2}(E)\right)=4 \text {, 而 } \operatorname{rank}\left(K_{2}\left(E_{\mathbb{Z}}\right)\right)=1 .
$$

进而, 分别得到 $K_{2}(E) \otimes \mathbb{Q}$ 和 $K_{2}\left(E_{\mathbb{Z}}\right) \otimes \mathbb{Q}$ 的一组基.

注记 4 对于文献 [7] 中的椭圆曲线 $E: 210 B 5,570 L 2,714 I_{1}, 870 I_{2}, 930 O_{4}$, 由定理 3.10 我们同样可以证明 $\operatorname{rank}\left(K_{2}(E)\right) \geqslant 3$. 


\section{4 引理 3.3-3.6 的证明}

引理 3.3 的证明 首先, 由定义 (1) 式或引理 2.4 , 易见 $\varepsilon(r, 0, i y) \in \mathbb{R}$.

令

$$
\gamma=\left(\begin{array}{cc}
0 & -1 \\
1 & 0
\end{array}\right) \in S L_{2}(\mathbb{Z})
$$

由引理 2.1(iii), 有

$$
\varepsilon(r, 0, i y)=\varepsilon\left(r, 0, \gamma \cdot\left(i y^{-1}\right)\right)=i y^{-3} \varepsilon\left(0, r, i y^{-1}\right) .
$$

因此对任意 $s \in\left(0, \frac{1}{2}\right)$ 和 $y>0$, 有 $\operatorname{Re} \varepsilon(0, s, i y)=0$. 所以只需证明对任意 $s \in\left(0, \frac{1}{2}\right)$ 和 $y>0$, $\operatorname{Im} \varepsilon(0, s, i y)$ 是一个正实数.

由引理 2.4, 有 $\operatorname{Im} \varepsilon(0, s, i y)=4 \pi^{3} B(s)-\pi^{2} y^{-1} A$, 其中

$$
A=\sum_{m \neq 0} \frac{1}{|m|} \sum_{n}(n-s) e^{-2 \pi y|n-s||m|}, \quad y>0 .
$$

令 $q:=e^{-2 \pi y|m|}, m \neq 0$, 则 $0<q<1$. 从而上式的内和为

$$
\begin{aligned}
\sum_{n}(n-s) q^{|n-s|} & =\sum_{n=1}^{\infty}(n-s) q^{|n-s|}+\sum_{n=1}^{\infty}((1-n)-s) q^{|(1-n)-s|} \\
& =\sum_{n=1}^{\infty}\left((n-s) q^{n-s}-(n-(1-s)) q^{n-(1-s)}\right) \\
& =q \frac{d}{d q} \sum_{n=1}^{\infty}\left(q^{n-s}-q^{n-(1-s)}\right),
\end{aligned}
$$

又

$$
\sum_{n=1}^{\infty}\left(q^{n-s}-q^{n-(1-s)}\right)=\left(q^{-s}-q^{-(1-s)}\right) \sum_{n=1}^{\infty} q^{n}=\frac{q^{1-s}-q^{s}}{1-q}
$$

因此

$$
\frac{d}{d q}\left(\frac{q^{1-s}-q^{s}}{1-q}\right)=\frac{1}{(1-q)^{2}}\left((1-s) q^{-s}-(1-s) q^{s}+s q^{1-s}-s q^{-(1-s)}\right)
$$

记

$$
D(q):=(1-s) q^{-s}-(1-s) q^{s}+s q^{1-s}-s q^{-(1-s)},
$$

则

$$
D^{\prime}(q)=s(1-s)\left(q^{-s}+q^{-(2-s)}-q^{-(1+s)}-q^{-(1-s)}\right) .
$$

令

$$
H(s):=q^{-s}+q^{-(2-s)} .
$$

则对 $0<q<1,0<s<\frac{1}{2}$, 有

$$
H^{\prime}(s)=q^{-s} \ln q^{-1}-q^{-(2-s)} \ln q^{-1}<0 .
$$

因此对任意 $s \in\left(0, \frac{1}{2}\right)$, 有

$$
D^{\prime}(q)=s(1-s)[H(s)-H(1-s)]=s(1-s) H^{\prime}\left(s_{0}\right)(2 s-1)>0,
$$


其中 $s_{0} \in(s, 1-s)$. 由于对 $0<q<1, D(q)$ 是连续的, 并且 $D(1)=0$, 从而对任意 $0<q<1$, 有 $D(q)<0$. 因此

$$
A=\sum_{m \neq 0} \frac{q}{|m|(1-q)^{2}} D(q)<0
$$

又对 $s \in\left(0, \frac{1}{2}\right)$,

$$
B(s)=\frac{1}{3} s^{3}-\frac{1}{2} s^{2}+\frac{1}{6} s=\frac{1}{3} s\left(s-\frac{1}{2}\right)(s-1)>0 .
$$

故

$$
\operatorname{Im} \varepsilon(0, s, i y)=4 \pi^{3} B(s)-\pi^{2} y^{-1} A>0 .
$$

引理 3.4 的证明 由引理 2.4, 对 $y>0$, 有

$$
\begin{aligned}
\operatorname{Im} \varepsilon\left(\frac{1}{4}, \frac{1}{2}, i y\right)= & 4 \pi^{3} B\left(\frac{1}{2}\right)-\pi^{2} y^{-1} \sum_{m \neq 0} \sum_{n} \frac{n-\frac{1}{2}}{|m|} e^{2 \pi i\left[\frac{m}{4}+i y\left|n-\frac{1}{2}\right||m|\right]} \\
= & -\pi^{2} y^{-1} \sum_{m \neq 0} \sum_{n} \frac{n-\frac{1}{2}}{|m|} e^{\frac{i m \pi}{2}} e^{-2 \pi y\left|n-\frac{1}{2}\right||m|} \\
= & \pi^{2} y^{-1} \sum_{m \neq 0} \frac{\frac{1}{2}}{|m|} e^{\frac{i m \pi}{2}} e^{-\pi y|m|} \\
& -\pi^{2} y^{-1} \sum_{m \neq 0} \sum_{n=1}^{\infty} \frac{n-\frac{1}{2}}{|m|} e^{\frac{i m \pi}{2}} e^{-2 \pi y\left(n-\frac{1}{2}\right)|m|} \\
& +\pi^{2} y^{-1} \sum_{m \neq 0} \sum_{n=1}^{\infty} \frac{n+\frac{1}{2}}{|m|} e^{\frac{i m \pi}{2}} e^{-2 \pi y\left(n+\frac{1}{2}\right)|m|} \\
= & 0 .
\end{aligned}
$$

因此对任意 $y>0, \varepsilon\left(\frac{1}{4}, \frac{1}{2}, i y\right)$ 是一个实数.

对 $n \geqslant 0, y>0$, 令 $q_{n}=e^{-(2 n+1) \pi y}$, 那么 $0<q_{n}<1$. 再由引理 2.4 , 有

$$
\begin{aligned}
\operatorname{Re} \varepsilon\left(\frac{1}{4}, \frac{1}{2}, i y\right)= & \frac{i \pi}{2} y^{-2} \sum_{m \neq 0} \sum_{n} \frac{1}{m|m|} e^{2 \pi i\left[\frac{m}{4}+i y\left|n-\frac{1}{2}\right||m|\right]} \\
& +i \pi^{2} y^{-1} \sum_{m \neq 0} \sum_{n} \frac{\left|n-\frac{1}{2}\right|}{m} e^{2 \pi i\left[\frac{m}{4}+i y\left|n-\frac{1}{2}\right||m|\right]} \\
= & -\frac{\pi}{2} y^{-2} \sum_{m \neq 0} \sum_{n} \frac{\sin \frac{m \pi}{2}}{m|m|} e^{-\pi y|2 n-1||m|} \\
& -\pi^{2} y^{-1} \sum_{m \neq 0} \sum_{n} \frac{\left|n-\frac{1}{2}\right| \sin \frac{m \pi}{2}}{m} e^{-\pi y|2 n-1||m|} \\
= & -2 \pi y^{-2} \sum_{m \geqslant 1} \sum_{n \geqslant 0} \frac{\sin \frac{m \pi}{2}}{m^{2}} q_{n}^{m} \\
& -2 \pi^{2} y^{-1} \sum_{m \geqslant 1} \sum_{n \geqslant 0} \frac{(2 n+1) \sin \frac{m \pi}{2}}{m} q_{n}^{m} \\
= & -2 \pi y^{-2} \sum_{n \geqslant 0} \sum_{m \geqslant 0}(-1)^{m} \frac{q_{n}^{2 m+1}}{(2 m+1)^{2}}
\end{aligned}
$$




$$
\begin{aligned}
& -2 \pi^{2} y^{-1} \sum_{n \geqslant 0}(2 n+1) \sum_{m \geqslant 0}(-1)^{m} \frac{q_{n}^{2 m+1}}{2 m+1} \\
& <0,
\end{aligned}
$$

上述不等式来源于下面显然的估计:

$$
\sum_{m \geqslant 0}(-1)^{m} \frac{q_{n}^{2 m+1}}{(2 m+1)^{2}}>0, \quad \sum_{m \geqslant 0}(-1)^{m} \frac{q_{n}^{2 m+1}}{2 m+1}>0 .
$$

因此对 $y>0$, 我们得到 $\varepsilon\left(\frac{1}{4}, \frac{1}{2}, i y\right)$ 是一个负实数.

注记 5 类似可以证明, 对 $y>0, r \in(0,1), \varepsilon\left(r, \frac{1}{2}, i y\right)$ 是一个实数.

引理 3.5 的证明 对 $r \in(0,1), y>0, \tau=\frac{1}{2}+i y$, 易证 $\varepsilon(r, 0, \tau)$ 是一个实数.

令

$$
\gamma=\left(\begin{array}{ll}
1 & -1 \\
2 & -1
\end{array}\right) \in S L_{2}(\mathbb{Z}),
$$

有

$$
\begin{aligned}
\varepsilon\left(r, 0, \frac{1}{2}+i y\right) & =\varepsilon\left(r, 0, \gamma \cdot\left(\frac{1}{2}+\frac{i}{4 y}\right)\right) \\
& =-\frac{i}{2^{3} y^{3}} \varepsilon\left(-r,-2 r, \frac{1}{2}+\frac{i}{4 y}\right) \\
& =\frac{i}{2^{3} y^{3}} \varepsilon\left(r, 2 r, \frac{1}{2}+\frac{i}{4 y}\right) .
\end{aligned}
$$

因此对 $y>0$, 有 $\operatorname{Re} \varepsilon\left(r, 2 r, \frac{1}{2}+\frac{i}{4 y}\right)=0$. 所以只需证明对 $y>0, \operatorname{Im} \varepsilon\left(r, 2 r, \frac{1}{2}+i y\right)$ 是一个正 实数.

由引理 2.4 有

$$
\begin{aligned}
\operatorname{Im} \varepsilon\left(r, 2 r, \frac{1}{2}+i y\right) & =4 \pi^{3} B(2 r)-\pi^{2} y^{-1} \sum_{m \neq 0} \sum_{n} \frac{n-2 r}{|m|} e^{2 \pi i\left[m\left(r-2 r \times \frac{1}{2}\right)+\frac{m n}{2}+i y|n-2 r||m|\right]} \\
& =4 \pi^{3} B(2 r)-\pi^{2} y^{-1} \sum_{m \neq 0} \sum_{n}(-1)^{m n} \frac{n-2 r}{|m|} e^{-2 \pi y|n-2 r||m|} .
\end{aligned}
$$

因此, 只需证明对 $y>0, s \in\left(0, \frac{1}{2}\right]$,

$$
4 \pi^{3} B(s)-\pi^{2} y^{-1} \sum_{m \neq 0} \sum_{n}(-1)^{m n} \frac{n-s}{|m|} e^{-2 \pi y|n-s||m|}=4 \pi^{3} B(s)-\pi^{2} y^{-1} M
$$

是一个正实数. 对于 $m \geqslant 1$, 令 $q_{m}=e^{-2 \pi y m}$, 有

$$
\begin{aligned}
M= & \sum_{m \neq 0} \sum_{n}(-1)^{m n} \frac{n-s}{|m|} e^{-2 \pi y|n-s||m|} \\
= & 2 \sum_{m=1}^{\infty} \sum_{n}(-1)^{m n} \frac{n-s}{m} q_{m}^{|n-s|} \\
= & -2 s \sum_{m=1}^{\infty} \frac{q_{m}^{s}}{m}+2 \sum_{m=1}^{\infty} \sum_{n=1}^{\infty}(-1)^{m n} \frac{n-s}{m} q_{m}^{n-s}-2 \sum_{m=1}^{\infty} \sum_{n=1}^{\infty}(-1)^{m n} \frac{n+s}{m} q_{m}^{n+s} \\
= & -2 s \sum_{m=1}^{\infty} \frac{q_{m}^{s}}{m}+2 \sum_{m=1}^{\infty} \sum_{n=1}^{\infty}(-1)^{m n} \frac{n}{m} q_{m}^{n}\left[q_{m}^{-s}-q_{m}^{s}\right] \\
& -2 s \sum_{m=1}^{\infty} \sum_{n=1}^{\infty}(-1)^{m n} \frac{1}{m} q_{m}^{n}\left[q_{m}^{-s}+q_{m}^{s}\right]
\end{aligned}
$$




$$
\begin{aligned}
& =-2 s \sum_{m=1}^{\infty} \frac{q_{m}^{s}}{m}+2 \sum_{m=1}^{\infty} \frac{(-1)^{m} q_{m}}{m\left(1-(-1)^{m} q_{m}\right)^{2}}\left[q_{m}^{-s}-q_{m}^{s}\right]-2 s \sum_{m=1}^{\infty} \frac{(-1)^{m} q_{m}}{m\left(1-(-1)^{m} q_{m}\right)}\left[q_{m}^{-s}+q_{m}^{s}\right] \\
& =2 \sum_{m=1}^{\infty}(-1)^{m} \frac{q_{m}}{m}\left\{\frac{q_{m}^{-s}-q_{m}^{s}}{\left(1-(-1)^{m} q_{m}\right)^{2}}-s \frac{q_{m}^{-s}+q_{m}^{s}+(-1)^{m} q_{m}^{(s-1)}\left(1-(-1)^{m} q_{m}\right)}{1-(-1)^{m} q_{m}}\right\} \\
& =2 \sum_{m=1}^{\infty}(-1)^{m} b_{m},
\end{aligned}
$$

其中

$$
b_{m}= \begin{cases}\frac{q_{m} D\left(q_{m}\right)}{m\left(1-q_{m}\right)^{2}} \leqslant 0, & m=2 k, k \geqslant 1, \\ \frac{s q_{m}^{s}-s q_{m}^{2-s}+(1-s) q_{m}^{1-s}-(1-s) q_{m}^{1+s}}{m\left(1+q_{m}\right)^{2}}>0, & m=2 k-1, k \geqslant 1 .\end{cases}
$$

这里的 $D(\cdot)$ 是引理 3.3 中定义的函数. 因此 $M$ 是一个负实数, 引理得证.

引理 3.6 的证明 类似引理 3.5 的证明过程可以证明, $\varepsilon\left(\frac{1}{3}, 0, \frac{1}{2}+i y\right)$ 是一个实数并且 有下面的等式:

其中

$$
\begin{aligned}
& \varepsilon\left(\frac{1}{3}, 0, \frac{1}{2}+i y\right)=\frac{i}{2^{3} y^{3}} \varepsilon\left(\frac{1}{3}, \frac{2}{3}, \frac{1}{2}+\frac{i}{4 y}\right), \\
& \varepsilon\left(\frac{1}{3}, \frac{2}{3}, \frac{1}{2}+i y\right)=-\varepsilon\left(\frac{2}{3}, \frac{1}{3}, \frac{1}{2}+i y\right), \\
& \operatorname{Im} \varepsilon\left(\frac{2}{3}, \frac{1}{3}, \frac{1}{2}+i y\right)=\frac{4 \pi^{3}}{3^{4}}-\frac{2 \pi^{2}}{3} \sum_{m=1}^{\infty}(-1)^{m-1} c_{m}(y),
\end{aligned}
$$

$$
c_{m}(y)= \begin{cases}\frac{e^{\frac{10 \pi y m}{3}}-2 e^{\frac{8 \pi y m}{3}}+2 e^{\frac{4 \pi y m}{3}}-e^{\frac{2 \pi y m}{3}}}{m y\left(e^{2 \pi y m}-1\right)^{2}}>0, \quad m=2 k, k \geqslant 1, \\ \frac{e^{\frac{10 \pi y m}{3}}+2 e^{\frac{8 \pi y m}{3}}-2 e^{\frac{4 \pi y m}{3}}-e^{\frac{2 \pi y m}{3}}}{m y\left(e^{2 \pi y m}+1\right)^{2}}>0, \quad m=2 k-1, k \geqslant 1 .\end{cases}
$$

对 $y>0, m \geqslant 1$, 令 $d_{m}(y)=c_{2 m-1}(y)-c_{2 m}(y)$.

断言 1 对 $y>0, m \geqslant 1$, 有 $d_{m}(y)>0$.

令 $t=\frac{2 y \pi}{3}, x=\frac{2(2 m-1) y \pi}{3}$, 则 $x=(2 m-1) t, x+t=2 m t$. 记 $w=e^{t}$, 有

$$
\begin{aligned}
d_{m}(y) & =\frac{2 \pi}{3}\left[\frac{e^{5 x}+2 e^{4 x}-2 e^{2 x}-e^{x}}{x\left(e^{3 x}+1\right)^{2}}-\frac{e^{5(x+t)}-2 e^{4(x+t)}+2 e^{2(x+t)}-e^{x+t}}{(x+t)\left(e^{3(x+t)}-1\right)^{2}}\right] \\
& =\frac{2 \pi}{3}\left[\frac{e^{x}\left(e^{2 x}-1\right)}{x\left(e^{2 x}-e^{x}+1\right)^{2}}-\frac{e^{x+t}\left(e^{2(x+t)}-1\right)}{(x+t)\left(e^{2(x+t)}+e^{x+t}+1\right)^{2}}\right] \\
& =\frac{2 \pi}{3}\left[\frac{w^{2 m-1}\left(w^{2(2 m-1)}-1\right)}{(2 m-1) t\left(w^{2(2 m-1)}-w^{2 m-1}+1\right)^{2}}-\frac{w^{2 m}\left(w^{4 m}-1\right)}{2 m t\left(w^{4 m}+w^{2 m}+1\right)^{2}}\right] .
\end{aligned}
$$

对 $m \geqslant 1, w>1$, 令

$$
\begin{aligned}
F_{m}(w)= & 2 m\left(w^{2(2 m-1)}-1\right)\left(w^{4 m}+w^{2 m}+1\right)^{2} \\
& -(2 m-1) w\left(w^{4 m}-1\right)\left(w^{2(2 m-1)}-w^{2 m-1}+1\right)^{2} .
\end{aligned}
$$

则

$$
F_{m}(w)=2 m w^{12 m-2}-(2 m-1) w^{12 m-3}+(8 m-2) w^{10 m-2}-2 m w^{8 m}
$$




$$
\begin{aligned}
& -(6 m-3) w^{8 m-1}+6 m w^{8 m-2}+(2 m-1) w^{8 m-3}-2 w^{6 m}+2 w^{6 m-2} \\
& -(2 m-1) w^{4 m+1}-6 m w^{4 m}+(6 m-3) w^{4 m-1}+2 m w^{4 m-2} \\
& -(8 m-2) w^{2 m}+(2 m-1) w-2 m .
\end{aligned}
$$

又当 $w>1, m \geqslant 1$ 时, 我们有下列不等式

$$
\begin{aligned}
& 2 m w^{12 m-2}-(2 m-1) w^{12 m-3}>w^{12 m-3}, \\
& (8 m-2) w^{10 m-2}-2 m w^{8 m}-(6 m-3) w^{8 m-1}>w^{8 m-1}, \\
& 6 m w^{8 m-2}-6 m w^{4 m}>0, \\
& (2 m-1) w^{8 m-3}-2 w^{6 m}+2 w^{6 m-2}-(2 m-1) w^{4 m+1}>0, \\
& (6 m-3) w^{4 m-1}+2 m w^{4 m-2}-(8 m-2) w^{2 m}>-w^{2 m}, \\
& (2 m-1) w-2 m>-1 .
\end{aligned}
$$

因此

$$
F_{m}(w)>w^{12 m-3}+w^{8 m-1}-w^{2 m}-1>0 .
$$

从而对 $m \geqslant 1, y>0$, 有 $d_{m}(y)>0$.

断言 2 对于 $m \geqslant 1, y \in(0, \infty), d_{m}(y)$ 是关于 $y$ 的递降函数.

对 $m \geqslant 1, y>0$, 令

则

$$
a=\frac{2 \pi(2 m-1)}{3}, \quad b=\frac{4 m \pi}{3}, g(y)=\frac{3 d_{m}(y)}{2 \pi} .
$$

$$
\begin{aligned}
g^{\prime}(y)= & \frac{a y\left(e^{3 a y}+1\right)\left(5 e^{5 a y}+8 e^{4 a y}-4 e^{2 a y}-e^{a y}\right)-\left[e^{5 a y}+2 e^{4 a y}-2 e^{2 a y}-e^{a y}\right]\left[(6 a y+1) e^{3 a y}+1\right]}{a y^{2}\left(e^{3 a y}+1\right)^{3}} \\
& -\frac{b y\left(e^{3 b y}-1\right)\left(5 e^{5 b y}-8 e^{4 b y}+4 e^{2 b y}-e^{b y}\right)-\left[e^{5 b y}-2 e^{4 b y}+2 e^{2 b y}-e^{b y}\right]\left[(6 b y+1) e^{3 b y}-1\right]}{b y^{2}\left(e^{3 b y}-1\right)^{3}} .
\end{aligned}
$$

令

$$
x=a y, \quad t=\frac{2 \pi y}{3}
$$

和

$$
\begin{aligned}
F(t)= & x\left(e^{3 x}+1\right)^{3}\left\{(x+t)\left(e^{3(x+t)}-1\right)\left(5 e^{5(x+t)}-8 e^{4(x+t)}+4 e^{2(x+t)}-e^{x+t}\right)\right. \\
& \left.-\left[e^{5(x+t)}-2 e^{4(x+t)}+2 e^{2(x+t)}-e^{(x+t)}\right]\left[(6(x+t)+1) e^{3(x+t)}-1\right]\right\} \\
& -(x+t)\left(e^{3(x+t)}+1\right)^{3}\left\{x\left(e^{3 x}+1\right)\left(5 e^{5 x}+8 e^{4 x}-4 e^{2 x}-e^{x}\right)\right. \\
& \left.-\left[e^{5 x}+2 e^{4 x}-2 e^{2 x}-e^{x}\right]\left[(6 x+1) e^{3 x}+1\right]\right\} .
\end{aligned}
$$

类似于断言 1 的证明, 可以证明对 $x \geqslant t>0, F(t)>0$. 因此 $g^{\prime}(y)<0$. 从而, 对 $m \geqslant 1$, $y>0, d_{m}(y)=\frac{2 \pi}{3} g(y)$ 是关于 $y$ 的递降函数.

所以对 $y>0$,

$$
\operatorname{Im} \varepsilon\left(\frac{2}{3}, \frac{1}{3}, \frac{1}{2}+i y\right)=\frac{4 \pi^{3}}{3^{4}}-\frac{2 \pi^{2}}{3} \sum_{m=1}^{\infty} d_{m}(y)
$$

是关于 $y$ 的递增函数. 另一方面, 易证当 $y$ 充分大时, $\operatorname{Im} \varepsilon\left(\frac{2}{3}, \frac{1}{3}, \frac{1}{2}+i y\right)>0$, 当 $y$ 充分小时, $\operatorname{Im} \varepsilon\left(\frac{2}{3}, \frac{1}{3}, \frac{1}{2}+i y\right)<0$. 
因此 $\varepsilon\left(\frac{1}{3}, 0, \frac{1}{2}+i y\right)$ 在区间 $(0, \infty)$ 内只有一个零点. 这样我们就完成了引理 3.6 的证明.

致谢＼cjkstart作者非常感谢审稿人仔细的审稿并提出宝贵的意见, 文中引理 3.6 就是在审稿人的 提议下给出的简化证明.

参考文献

1 Bloch S, Grayson D. $K_{2}$ of Elliptic Curves and Values of L-functions: Computer Calculations. Contemporary Mathematics 55, Providence, RI: Amer Math Soc, 1983, 79-88

2 Birch B J, Kuyk W. Modular Functions of One Variable IV. Lecture Notes in Mathematics 476 . New York-Berlin-Heidelberg: Springer-Verlag, 1975

3 Ross R. $K_{2}$ of elliptic curves with sufficient torsion over $\mathbb{Q}$. Comp Math, 81: 211-221 (1992)

4 Beilinson A A. Higher regulators and values of L-functions of curves. Funct Anal Appl, 14: 116-118 (1980)

5 Bloch S. Higher Regulators, Algebraic K-Theory, and Zeta Functions of Elliptic Curves. CRM MonographSeries, Vol 11. Providence, RI: Amer Math Soc, 2000

6 Silverman J H. Advanced Topics in the Arithmetic of Elliptic Curves. New York-Berlin-Heidelberg-Tokyo: Springer-Verlag, 1994

7 Cremona J. Algorithms for Modular Elliptic Curves. Cambridge: Cambridge University Press, 1997

8 Knapp A W. Elliptic Curves. Princeton: Princeton University Press, 1992

9 Lang S. Elliptic Functions, GTM(112). New York-Berlin-Heidelberg: Springer-Verlag, 1987

10 Ramakrishnan D, Regulators, Algebraic Cycles, and Values of L-functions. In: Contemporary Mathematics, 83, Providence, RI: Amer Math Soc, 1989, 183-310

11 Silverman J H. The arithmetic of elliptic curves. New York-Berlin-Heidelberg-Tokyo: Springer-Verlag, 1986

12 Silverman J H. Computing heights on elliptic curves. Math Comp, 51: 339-358 (1988) 